\title{
Mutations in the P3 Protein of Soybean mosaic virus G2 Isolates Determine Virulence on Rsv4-Genotype Soybean
}

\author{
R. V. Chowda-Reddy, ${ }^{1}$ Haiyue Sun, ${ }^{1,2}$ Hongyan Chen, ${ }^{1,2}$ Vaino Poysa, ${ }^{3}$ Hong Ling, ${ }^{4}$ Mark Gijzen, ${ }^{1}$ and \\ Aiming Wang ${ }^{1}$ \\ ${ }^{1}$ Southern Crop Protection and Food Research Centre, Agriculture and Agri-Food Canada, 1391 Sandford St., London, \\ Ontario, N5V 4T3, Canada; ${ }^{2}$ Department of Biology, Biological \& Geological Building, The University of Western Ontario, \\ London, Ontario, N6A 5B7, Canada; ${ }^{3}$ Greenhouse and Processing Crops Research Centre, Agriculture and Agri-Food \\ Canada, 2585 County Rd. 20, Harrow, Ontario, NOR 1G0, Canada; ${ }^{4}$ Department of Biochemistry, Medical Sciences \\ Building, The University of Western Ontario, London, Ontario, N6A 5C1, Canada
}

Submitted 14 July 2010. Accepted 24 August 2010.

Two Soybean mosaic virus (SMV) G2 isolates, L and L-RB, sharing high-sequence similarly but differing in ability to break $R s v 4$-mediated resistance in soybean, were investigated. Infectious clones corresponding to these two isolates and their chimeric clones resulting from swapping different regions of genomic cDNA between $L$ and $L-R B$ were constructed. Only L-RB or chimeras containing the middle fragment of L-RB cDNA showed virulence on Rsv4-genotype soybean. Sequence comparison analysis revealed that the middle genomic region of $L$ and $L-R B$ encodes four different amino acids. Point mutagenesis demonstrated that a single amino acid substitution (Q1033K) in the $P 3$ protein determined virulence toward $R s v 4$ resistance. In addition, six new SMV Rsv4 resistance-breaking isolates, variants of the second passage on Williams 82 infected with the chimeras or mutants noninfectious on soybean carrying $R s v 4$, were obtained. Sequencing data indicated that these new isolates contain either the Q1033K mutation or a new substitution (G1054R) in P3. Site-directed mutagenesis confirmed the virulence role of the G1054R mutation on Rsv4genotype soybean. Taken together, these data suggest that P3 of the SMV G2 strain is an avirulent determinant for Rsv4 and one single nucleotide mutation in P3 may be sufficient to compromise its elicitor function.

Plant viruses are obligate intracellular parasites that infect many agriculturally important crops and cause a significant loss in crop yields worldwide every year. Utilization of genetic resistance has been considered the most effective and environmentally sustainable means to manage viral diseases. Previously, approximately a dozen viral resistance $(R)$ genes have been reported (Maule et al. 2007); most such genes function through direct or indirect interactions with a viral protein (avirulence factor) encoded by corresponding viral pathogens (Janzac et al. 2010; Kim et al. 2010). To evade $R$-mediated rec-

Corresponding author: Aiming Wang; Telephone: +1 5194571470 ext 313; Fax: +1 519 4573997; E-mail: Aiming.Wang@ AGR.GC.CA

Nucleotide sequence data is available in the GenBank database under accession numbers HQ166265 and HQ166266.

* The $\boldsymbol{e}$-Xtra logo stands for "electronic extra" and indicates that two supplementary figures are published online. ognition, plant viruses have evolved a survival strategy by generating viral quasi-species, mainly through RNA recombination, and genomic RNA mutations due to the high error rate of the viral RNA-dependent RNA polymerase (García-Arenal et al. 2003). The genetic variability of the quasi-species and a selection force that acts on this variability can lead to the emergence of resistance-breaking isolates (Gagarinova et al. 2008a and $b$ ).

The genus Potyvirus is the largest and most agriculturally important virus group, representing approximately $30 \%$ of known plant viruses (Berger et al. 2005). Potyviruses have a monopartite, single-stranded, positive-sense RNA genome of approximately 9.6-kb nucleotides with VPg (genome-linked viral protein) covalently linked to its $5^{\prime}$ end and a poly-A tail at the $3^{\prime}$ end (Fig. 1). The viral genome contains a single open reading frame (ORF) encoding a large polyprotein of approximately $350 \mathrm{kDa}$ that is ultimately cleaved into 10 end protein products (Jayaram et al. 1992; Urcuqui-Inchima et al. 2001). The 10 mature proteins, beginning from the $\mathrm{N}$ terminus of the polyprotein, are P1, HC-Pro, P3, 6K1, CI, 6K2, NIa (which is further cleaved into the NIa-VPg protein or VPg and the NIaPro), Nib, and CP (Fig. 1). In addition, a translational frameshift on the P3 cistron leads to the production of an additional protein, termed P3N-PIPO (Chung et al. 2008), which is essential for potyvirus cell-to-cell movement (Wei et al. $2010 \mathrm{~b})$. Of these 11 mature viral proteins, the $6 \mathrm{~K} 2$ protein $(6 \mathrm{~K}$ or 6-kDa) and the $\mathrm{P} 3$ protein are the only two viral membrane proteins (Eiamtanasate et al. 2007; Restrepo-Hartwig and Carrington 1994). The $6 \mathrm{~K} 2$ protein has been shown to play a pivotal role in the formation of the virus replication complex (Schaad et al. 1997; Wei and Wang 2008; Wei et al. 2010a). The P3 protein containing two hydrophobic domains located at the $\mathrm{N}$ and the $\mathrm{C}$ terminus, respectively, is relatively poorly studied. Based on its subcellular distribution, we have suggested that P3 may play dual roles in potyvirus movement and replication (Cui et al. 2010).

Soybean mosaic virus (SMV), a Potyvirus sp., is the most common viral pathogen of legumes, with soybean as the primary agronomic host (Gunduz et al. 2004). The seedborne, aphid-transmitted virus causes severe yield losses and seed quality deterioration worldwide. To date, numerous SMV isolates have been reported. In the United States, SMV is grouped into seven strains, G1 through G7, based on responses on two susceptible and six resistant soybean cultivars (Cho and 
Goodman 1979). Extensive screening for soybean germplasm resistant to SMV results in identification of three independent resistance loci (i.e., $R s v 1, R s v 3$, and $R s v 4$ ) with different SMV strain specificities (Gunduz et al. 2002; Hayes et al. 2000; Liao et al. 2002; Zheng et al. 2005). Rsv1, Rsv3, and $R s v 4$ are all dominant genes that have been mapped to molecular linkage groups F, B2, and D1b, respectively (Gore et al. 2002; Hayes et al. 2000; Jeong et al. 2002). Rsv2, initially assigned to the resistance gene in OX670, was dropped after OX670 had been shown to actually possess two resistance genes, $R s v 1$ and $R s v 3$ (Gunduz et al. 2001). Rsv1, found in PI96983 and also named $R s v$, confers resistance to the SMV strains G1 to G6 but not to G7, whereas Rsv3-carrying soybean is resistant to the SMV strains G5 to G7 but susceptible to G1 to G4 (Buss et al. 1999). Rsv4 is the only resistance gene that shows resistance to all seven strains (Gunduz et al. 2004). The mechanism of $R s v 4$ resistance seems different from that of $R s v 1$ and $R s v 3$ because it is not associated with extreme resistance or hypersensitive responses (Gunduz et al. 2004). All of these reflect the complex interactions of SMV and resistance genes.

Recently, several naturally occurring SMV isolates capable of overcoming resistance genes at all three soybean resistance loci $(R s v 1, R s v 3$, and $R s v 4$ ) have been found in Korea (Choi et al. 2005). More recently, we have also reported identification of a naturally occurring $R s v 4$ resistance-breaking isolate (LRB) and its closely related nonresistance-breaking avirulent isolate (L) in Canada (Gagarinova et al. 2008a and b). In this study, we investigated the genetic virulence determinant of SMV L-RB on Rsv4-genotype soybean.

\section{RESULTS}

Construction of infectious clones of SMV $L$ and $L-R B$ isolates.

$\mathrm{L}$ and L-RB are two naturally occurring SMV isolates that are closely related but differ in their ability to overcome Rsv4 resistance (Gagarinova et al. 2008a). Both isolates have a 9,617-nucleotide genome (including a 32-A tail). For cloning convenience, the full-length cDNA was divided into three fragments (i.e., N, M, and C) (Fig. 1) These three fragments were sequentially inserted into a modified pBR322 vector (Fig. 1). All the inserted cDNA was completely sequenced to ensure that no mutations were introduced. The resulting clones, pSMV-L and pSMV-LRB, contain the full-length cDNA of isolates $\mathrm{L}$ and $\mathrm{L}-\mathrm{RB}$, respectively.

To test whether pSMV-L and pSMV-LRB are infectious, 2week-old seedlings of soybean-susceptible indicator cv. Williams 82 were bombarded with plasmid DNA. Fifteen days postbombardment, all the four soybean seedlings bombarded with either pSMV-L or pSMV-LRB showed typical SMV symptoms as previously described (Gagarinova et al. 2008a). enzyme-linked immunosorbent assay (ELISA) and reversetranscription polymerase chain reaction (RT-PCR) analysis confirmed the presence of SMV (data not shown). Symptomatic leaves from Williams 82 plants were used as an inoculum for a pathogenicity test. Williams 82 (rsv, carrying no resistance gene) and five resistant cultivars or accessions, including PI96983 (Rsv1), L78-379 (Rsv1), L29 (Rsv3), Suweon97 (Rsv1-h), and V94-5152 $(R s v 4)$, carrying an SMV resistance gene (indicated

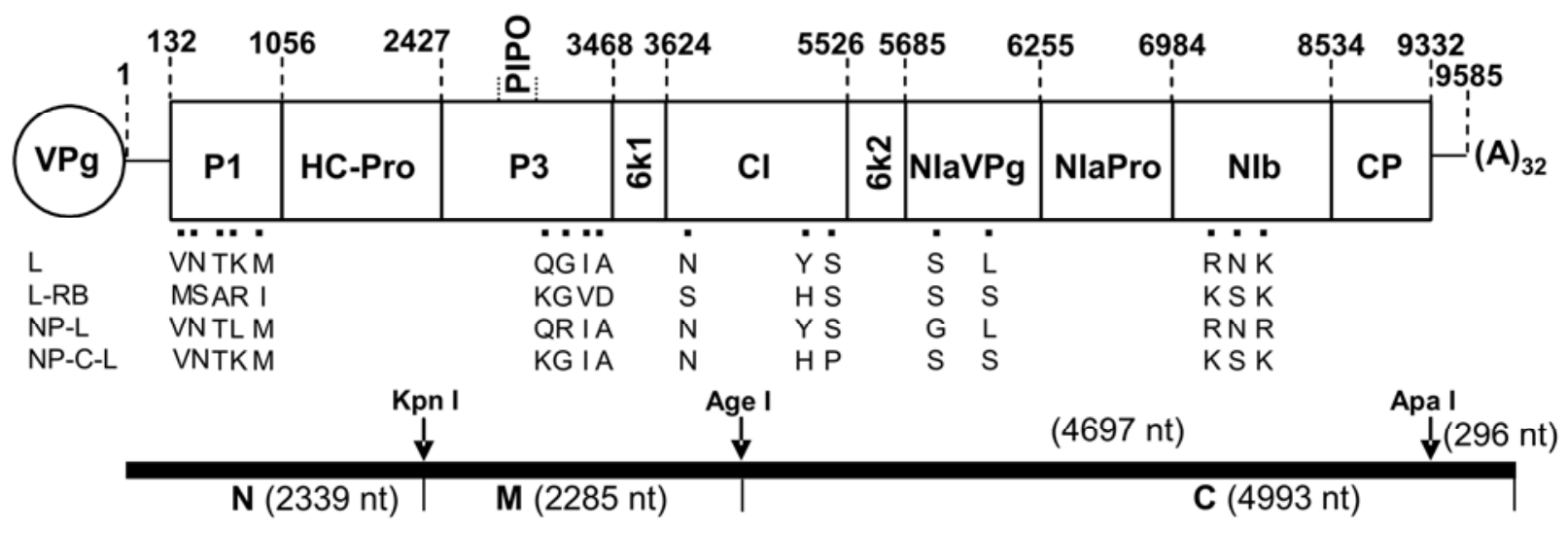

Fig. 1. Construction of infectious Soybean mosaic virus (SMV) clones. The backbone vector is a modified pBR322 vector containing a Cauliflower mosaic virus $35 \mathrm{~S}$ promoter and the NOS terminator. The SMV full-length cDNA consists of 9,617 nucleotides (nt), including a 131 -nt $5^{\prime}$ untranslational region, a 256-nt 3' untranslational region, and a 32-A tail. For cloning convenience, the cDNA is divided into three fragments: N, M, and C. The SMV L and L-RB isolates, designated L and L-RB, respectively, were obtained previously (Gagarinova et al. 2008a) (GenBank accession nos. EU871724 and EU871725). Two new SMV isolates designated NP-C-L and NP-L were obtained in this study with GenBank accession nos. HQ166265 and HQ166266, respectively. Comparison of these four isolates revealed a total of 17 amino acid mismatches, indicated as solid small squares. Their exact amino acid positions (amino acid identities) in four isolates (L, L-RB, NP-L, and NP-C-L) are 43 (V/M/V/V), 52 (N/S/N/N), 137 (T/A/T/T), 156 (K/R/L/K), 252 (M/I/M/M), 1,033 (Q/K/Q/K), 1,054 (G/G/R/G), 1,081 (I/V/I/I), 1,108 (A/D/A/A), 1,171 (N/S/N/S), 1,677 (Y/H/Y/H), 1,685 (S/S/S/P), 1,804 (S/S/G/S), $2,029(\mathrm{~L} / \mathrm{S} / \mathrm{L} / \mathrm{S}), 2,672(\mathrm{R} / \mathrm{K} / \mathrm{R} / \mathrm{K}), 2,744(\mathrm{~N} / \mathrm{S} / \mathrm{N} / \mathrm{S})$, and 2,824 $(\mathrm{K} / \mathrm{K} / \mathrm{R} / \mathrm{K})$. For clarity, the relative sizes of the mature proteins or cDNA fragments are not drawn to scale.

Table 1. Response of different soybean cultivars inoculated with pSMV-L- or pSMV-LRB-derived inoculuma

\begin{tabular}{lllll}
\hline Cultivars & Resistant allele & \multicolumn{1}{c}{ Relationship with SMV strains } & pSMV-L & pSMV-LRB \\
\hline Williams82 & None & Susceptible indicator & Severe mosaic & Severe mosaic \\
PI 96983 & Rsv1 & Resistant to G1-G6, susceptible to G7 & - & - \\
L78-379 & Rsv1 & Williams 82 isoline with Rsv1 & - & Severe mosaic \\
L29 & Rsv3 & Resistant to G5-G7, susceptible to G1-G4 & Severe mosaic & - \\
Suweon 97 & Rsv1-h & Resistant to G1-G7 & - & Severe mosaic/chlorosis \\
V94-5152 & Rsv4 & Resistant to G1-G7 & - & \\
\hline
\end{tabular}

${ }^{a}$ Soybean plants were observed 15 days postinoculation with inocula derived from infectious clones pSMV-L and pSMV-LRB; - indicates that plants were healthy and enzyme-linked immunosorbent assay and reverse-transcription polymerase chain reaction failed to detect the presence of Soybean mosaic virus (SMV). 
in italics), were inoculated with pSMV-L- or pSMV-LRBderived inocula. The pSMV-L-derived inoculum infected Williams 82 and L29 but failed to infect PI 96983, L78-379, Suweon 97, and V94-5152 (Table 1; Fig. 2A). In addition to Williams 82 and L29, the pSMV-LRB-derived inoculum also infected V94-5152 carrying Rsv4 (Table 1; Fig. 2B). Thus, the differential response of V94-5152 to pSMV-L- or pSMV-LRBderived inocula was consistent with that to L or L-RB isolates (Gagarinova et al. 2008a), suggesting that pSMV-L and pSMV-LRB are the infectious clones resembling SMV isolates $\mathrm{L}$ and L-RB, respectively.

\section{Determination of the SMV L-RB genomic fragment responsible for breaking $R s v 4$ resistance.}

To map which genomic region is responsible for breaking $R s v 4$ resistance, a number of chimeric clones resulting from swapping N, M, or C cDNA fragments of pSMV-L and pSMVLRB were generated. All the swapping clones bombarded onto Williams 82 ( $r s v)$ carrying no resistance gene showed infectivity. Subsequent pathogenicity tests confirmed that all chimeras were infectious on Williams 82 (Fig. 3). In addition, all chimeras also infected L29 that carries the Rsv3 resistance gene (Fig. 3). The symptoms induced by these chimeras were very similar among soybean plants, and upper trifoliate leaves usually showed more severe symptoms (Fig. 2). These data indicate that all chimeras retain infectivity characteristic of the SMV G2 group. However, some chimeric clones showed differential infectivity on V94-5152 carrying the Rsv4 resistance gene (Fig. 3). When the $M$ fragment of the $L$ isolate was substituted with that of L-RB, the resulting clone, $\mathrm{pSMV}-\mathrm{L}\left(\mathrm{M}_{\mathrm{LRB}}\right)$, gained virulence on V94-5152 (Figs. 2C and 3). The virulence of $\mathrm{L}$ $\mathrm{RB}$ on V94-5152 was lost if the $\mathrm{M}$ region of $\mathrm{L}-\mathrm{RB}$ was replaced by that of L. All the other derivatives of pSMV-L or
pSMV-LRB resulting from swapping $\mathrm{N}$ or $\mathrm{C}$ fragments did not affect its own infectivity on V94-5152. Thus, the infectivity on $R s v 4$ soybean (breaking $R s v 4$ resistance) was conditional upon the presence of the M cDNA fragment of the L-RB isolate (Fig. 3). Taken together, these data suggest that the $M$ fragment of the L-RB isolate is responsible for breaking $R s v 4$ resistance.

\section{The point mutation of Q1033K within the $\mathrm{C}$-terminal membrane domain of $\mathrm{P3}$ is essential for breaking $R s v 4$ resistance.}

The $\mathrm{M}$ fragment of isolates $\mathrm{L}$ and L-RB consists of four amino acid mismatches (Fig. 1). The exact positions (identities) of these four amino acids in $\mathrm{L}$ and $\mathrm{L}-\mathrm{RB}$ are $1,033(\mathrm{Q} / \mathrm{K})$, $1,081(\mathrm{I} / \mathrm{V}), 1,108(\mathrm{~A} / \mathrm{D})$, and 1,171 (N/S), with the first three in the $\mathrm{P} 3$ protein and the last one in CI. To identify mutations essential for breaking $R s v 4$ resistance, site-directed mutagenesis was performed. Initially, two single mutations (i.e., Q1033K and I1081V) were introduced into pSMV-L to produce clones pSMV-L(Q1033K) and pSMV-L(I1081V). These two clones were bombarded into Williams 82 soybean. Pathogenicity tests revealed that both clones inherited infectivity of L on the Rsv3 cultivar (Fig. 4). However, they showed differential infectivity on V94-5152; the mutant containing Q1033K gained ability to overcome $R s v 4$ resistance (Fig. 4). Then, three double mutants-that is, pSMV-L(I1081V,A1108D), pSMV-L(I1081V,N1171S), and pSMV-L(Q1033K,I1081V)and three triple mutants-that is, pSMV-L(I1081V,A1108D, N1171S), pSMV-L(Q1033K,I1081V,N1171S), and pSMVL(Q1033K,I1081V,A1108D)—were generated. The double mutant containing Q1033K and I1081V and the triple mutant containing Q1033K, I1081V, and N1171S lost the ability to infect the susceptible indicator soybean plants (Fig. 4). Thus,
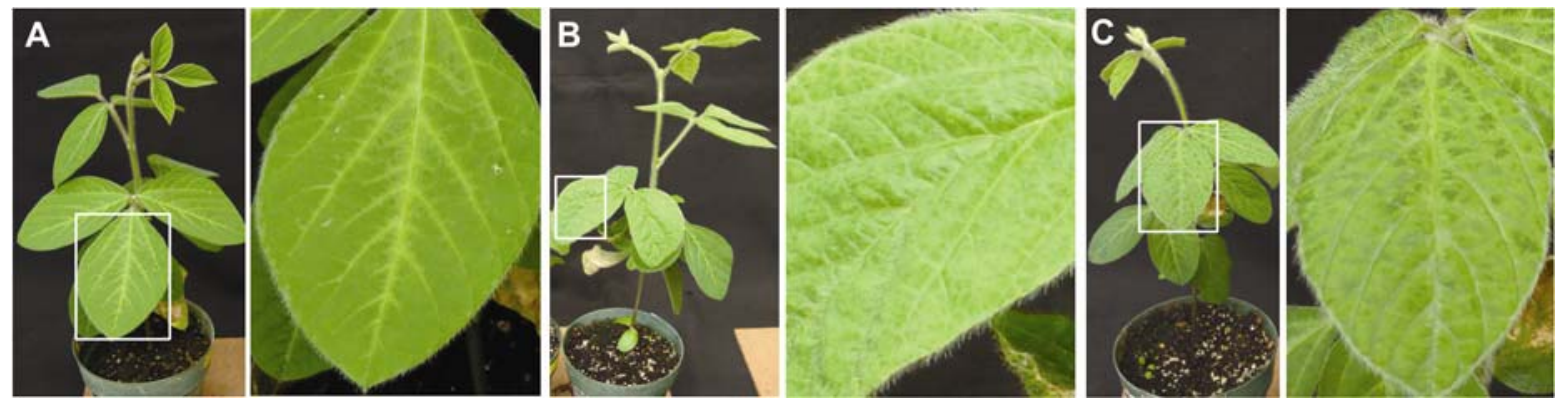

Fig. 2. Response of V94-5152 inoculated with the inocula derived from different Soybean mosaic virus (SMV) infectious clones. A, pSMV-L; B, pSMV-LRB; and $\mathbf{C}$, pSMV-L $\left(\mathrm{M}_{\mathrm{LRB}}\right)$. Fifteen days postbombardment, the soybean plants showed A, asymptomatic; $\mathbf{B}$, mosaic; and $\mathbf{C}$, severe mosaic. For better viewing, a selected portion in the rectangle is enlarged and shown to the right.

Clone Name
pSMV-L (wild-type)
pSMV-LRB (wild-type)
pSMV-L $\left(\mathrm{N}_{\mathrm{LRB}}\right)$
pSMV-L $\left(\mathrm{M}_{\mathrm{LRB}}\right)$
pSMV-L $\left(\mathrm{C}_{\mathrm{LRB}}\right)$
pSMV-LRB $\left(\mathrm{N}_{\mathrm{L}}\right)$
pSMV-LRB $\left(\mathrm{M}_{\mathrm{L}}\right)$
pSMV-LRB $\left(\mathrm{C}_{\mathrm{L}}\right)$

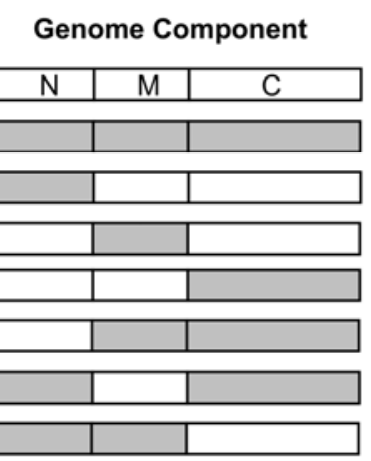

\begin{tabular}{ccccc}
\multicolumn{5}{c}{ Infectivity } \\
$r s v$ & $R s v 1$ & $R s v 1-h$ & $R s v 3$ & $R s v 4$ \\
+ & - & - & + & - \\
+ & - & - & + & + \\
+ & - & - & + & - \\
+ & - & - & + & + \\
+ & - & - & + & - \\
+ & - & - & + & + \\
+ & - & - & + & - \\
+ & - & - & + & +
\end{tabular}

Fig. 3. Identification of the cDNA fragment responsible for breaking Rsv4-mediated resistance using chimeric pSMV-L and pSMV-LRB infectious clones resulting from swapping the N, M, and C cDNA fragments containing an Soybean mosaic virus (SMV) resistance gene (indicated in italics). The infectivity of the chimeric clones is shown to the right. rsv, Williams 82 (carrying no resistance gene); Rsv1, PI96983 or L78-379; Rsv1-h, Suweon97; Rsv3, L29; Rsv4, V94-5152; -, negative; +, positive. 
these two clones were lethal mutants. A triple mutant that was created by introducing an A1108D mutation into the double mutant pSMV-L(Q1033K,I1081V) rescued the lethal double mutant and also gained the ability to break Rsv4 resistance (Fig. 4). No other double or triple mutants could infect V945152. Thus, the Q1033K substitution resulting from a single nucleotide mutation is essential for gaining the ability to infect V94-5152. To confirm this, a point mutation of K1033Q was made in pSMV-LRB. Indeed, the mutant lost the ability to infect Rsv4 soybean (Fig. 4). Taken together, these data suggest that the Q1033K mutation within the C-terminal membrane domain is responsible for L-RB to gain virulence on V94-5152.

\begin{tabular}{lccccc}
\multicolumn{1}{c}{ Clone names } & \multicolumn{5}{c}{ Infectivity } \\
pSMV-L (QGIAN, wild-type) & + & - & - & + & - \\
pSMV-LRB (KGVDS, wild-type) & + & - & - & + & + \\
pSMV-L(Q1033K) & + & - & - & + & + \\
pSMV-L(I1081V) & + & - & - & + & - \\
pSMV-L(I1081V,A1108D) & + & - & - & + & - \\
pSMV-L(I1081V,N1171S) & + & - & - & + & - \\
pSMV-L(I1081V,A1108D,N1171S) & + & - & - & + & - \\
pSMV-L(Q1033K,I1081V) & - & $\mathrm{n} / \mathrm{a}$ & $\mathrm{n} / \mathrm{a}$ & $\mathrm{n} / \mathrm{a}$ & $\mathrm{n} / \mathrm{a}$ \\
pSMV-L(Q1033K,I1081V,N1171S) & - & $\mathrm{n} / \mathrm{a}$ & $\mathrm{n} / \mathrm{a}$ & $\mathrm{n} / \mathrm{a}$ & $\mathrm{n} / \mathrm{a}$ \\
pSMV-L(Q1033K,I1081V,A1108D) & + & - & - & + & + \\
pSMV-LRB(K1033Q) & + & - & - & + & - \\
pSMV-L(G1054R) & + & - & - & + & +
\end{tabular}

Fig. 4. Identification of the amino acids encoded in the $M$ fragment responsible for breaking Rsv4-mediated resistance though point mutagenesis. The $\mathrm{M}$ fragment encodes the C-terminal portion of HC-Pro, the full length of $\mathrm{P} 3$ (including PIPO) and $6 \mathrm{~K} 1$, and the N-terminal portion of CI. There are four mismatches in the $\mathrm{M}$ fragment of $\mathrm{L}$ and $\mathrm{L}-\mathrm{RB}$ (showing in parentheses), with three in the C-terminus of $\mathrm{P} 3$ and one in the N-terminus of CI. The exact positions (identities) of these four amino acids in $\mathrm{L}$ and $\mathrm{L}-\mathrm{RB}$ are 1,033 (Q/K), 1,081 (I/V), 1,108 (A/D), and $1,171(\mathrm{~N} / \mathrm{S})$. In addition, a new mismatch at position 1,054 was found in the newly identified resistance-breaking isolate NP-L. Mutations are indicated in the clone name. For example, pSMV-L(Q1033K) indicates that the amino acid $\mathrm{Q}$ at position 1,033 in the $\mathrm{L}$ infectious clone was mutated to $\mathrm{K}$. The infectivity of the parental and mutant clones is shown to the right. rsv, Williams 82 (carrying no resistance gene); Rsv1, PI96983 or L78-379; Rsv1-h, Suweon97; Rsv3, L29; Rsv4, V94-5152; -, negative; +, positive.

\section{Identification of an alternative mutation (G1054R) near the $\mathrm{C}$-terminal membrane domain of $\mathrm{P} 3$ responsible for overcoming $R s v 4$ resistance.}

To obtain new resistance-breaking isolates, V94-5152 was mechanically inoculated with inocula from the second passage on Williams 82 soybean infected with all nine infectious clones (including mutants) that failed to infect V94-5152. After 35 days postinoculation, SMV symptoms were observed in newly emerging leaves of 6 soybean seedlings (out of 27 seedlings inoculated). Of these, three were from pSMV-L, two from pSMV-L $\left(\mathrm{C}_{\mathrm{LRB}}\right)$, and one from pSMV-L (I1081V,N1171S). Pathogenicity tests confirmed that these new SMV isolates were virulent on V94-5152. The M region of all the six isolates was sequenced. Comparison of deduced amino acid sequences with their corresponding parental clone sequences revealed the Q1033K mutation present in two isolates and a G1054R mutation in the other four isolates. Two representative new resistance-breaking isolates named NP-L derived from pSMV-L and NP-C-L from pSMV-L $\left(\mathrm{C}_{\mathrm{LRB}}\right)$, consisting of G1054R and Q1033K mutations, respectively, were completely sequenced. In comparison with their respective parental clones, isolates NP-C-L and NP-L have two and four amino acid substitutions, respectively (Fig. 1). Because the Q1033K mutation shown to be required for overcoming Rsv4 resistance is located within the C-terminal membrane domain, the G1054R mutation near the C-terminal membrane domain might also be essential for NP-L to gain virulence toward Rsv4. To test this possibility, pSMV-L was mutated to generate mutant pSMV-L(G1054R). Indeed, the virulence of pSMV-L(G1054R) to different soybean cultivars was the same as the L-RB isolate (Fig. 4). Thus, like the Q1033K mutation, a single amino acid G1054R substitution resulting from a single nucleotide mutation is also sufficient to gain virulence toward $R s v 4$ resistance.

\section{Modeling of the three-dimensional structures of the $P 3$ protein and its variants.}

Amino acid sequence alignments of the $\mathrm{P} 3$ proteins of SMV isolates L, L-RB, NP-C-L, and NP-L and other SMV isolates, including G5, G7d, G7H, G7, and N, showed that the positively charged amino acid $\mathrm{K}$ (position 1,033) or $\mathrm{R}$ (position 1,054 ) is unique to the resistance-breaking isolates (Supplementary Fig. S1). These two amino acids are located either within or near the P3 C-terminal membrane domain. To predict the three-dimensional structures of the $\mathrm{P} 3$ protein of the $\mathrm{L}$ isolate and its Q1033K and G1054R mutants, PHYRE analyses were performed (Kelley and Sternberg 2009). A Protein Data

\begin{abstract}
G5H 1025 ICIIFSLLVQMTSVLQGIVNTARRDKALLNGWKRKEDEEAVIHLYEMCEKMEGGHPSIEKFLDHVKGVRP 1094
G7d 1025 ICIIFSLLVQMTSVLQGIINTARRDKALLHSHKRREDEEAVIHLYEMCEKMEGGHPSVEGFLNHVRGVRP 1094

G7H 1025 ICIIFSLLVQMTSVLQGIVNTARRDKALLNGWKRKEDEEAVIHLYEMCEKMEGGHPSIEKFLDHVKGVRP 1094 G7 1025 ICIIFSLLVQMTSVLQGI INTARRDKALLHSHKRREDEEAVIHLYEMCEKMEGGHPSVEGFLNHVRGVRP 1094

L 1024 ICIIFSLLVQMTSVLQGIVNTARRDKALLSGWKRKEDEEAVIHLYEMCEKMEGGHPSIEKFLDHVKGVRP 1093

L-RB 1024 ICIIFSLLVKMTSVLQGIVNTARRDKALLSGWKRKEDEEAVIHLYEMCEKMEGGHPSVEKFLDHVKGVRP 1093

N 1024 ICIIFSLLVQMTSVLQGIVNTVRRDKALLSGWKRKEDEEAVIHLYEMCEKMEGGHPSIEKFLDHVKGVRP 1093

NP-L 1024 ICIIFSLLVQMTSVLQGIVNTARRDKALLSRWKRKEDEEAVIHLYEMCEKMEGGHPSIEKFLDHVKGVRP 1093

NP-C-L 1024 ICIIFSLLVKMTSVLQGIVNTARRDKALLSGWKRKEDEEAVIHLYEMCEKMEGGHPSIEKFLDHVKGVRP 1093

GM01g03890 529 KSMALVALLKLSSRFPSCSERIKEIIVQFKGSFVLELQQRAIEFNSIIAKHQNIRSTLVERMPVLDEATS 598

GM02g03800 529 KAMALVALLKLSSRFPSCSERIKEIIVQFKGSFVLELQQRAIEFSSIISKHQNIRSTLVERMPVLDEATY 598

GM08g39930 529 KAMALVALLKLSSRFPSCSERIREIIVQCKGNLVLELQQRSIEFNLI IAKHQNIRPTLVERMPVLDEVTF 598

AT1g60070 529 KAMALIALLKISSRFPSCSERVKSIIGQNKGSFVLELQQRSLEFSSVIQKHQNIRSSLVERMPVLDEATF 598

GM18g18490 610 ETMALVALLKLSSRFPSCSERIREIIVQCKGNLVLELQQRSIEFNLIIAKHQNIRHTLVERMPVLDEATF 679

Human AP-1 523 RGYALTAIMKLSTRFTCTVNRIKKVVSIYGSSIDVELQQRAVEYNALFKKYDHMRSALLERMPVMEKVTT 592
\end{abstract}

Fig. 5. Amino acid alignments of the region of Soybean mosaic virus (SMV) P3 involved in breaking Rsv4-mediated resistance with the homologous regions of human adaptor protein (AP)-1 and soybean and Arabidopsis homologs. The alignment was constructed by Genedoc. GenBank accession numbers of SMV isolates are shown in parenthesis: G5H (FJ807701), G7d (AY216986), G7H (AY294045), G7 (AY216010), L (EU871724), L-RB (EU871725), N (D00507), NP-L (HQ166266), and NP-C-L (HQ166265). GM01g03890, GM02g03800, and GM18g18490 are from soybean; AT1g60070 from Arabidopsis; and human AP1 served as a template. 
Bank (PDB) homolog search revealed a PDB template, 1w63:K (i.e., the human adaptor protein [AP-1] complex subunit gamma-1). This template protein shares $8 \%$ identity with $\mathrm{P} 3$ at a precision of $80 \%$. Because soybean is the natural host to SMV and Arabidopsis is the best-characterized model plant species, BLAST searches were carried out using Phytozome, a tool for green plant comparative genomics, against soybean and Arabidopsis protein sequences to identify the proteins that are homologous to the human AP-1. The searches identified four soybean AP-1-like proteins (GM01g03890, GM02g03800, GM08g39930, and GM18g18490) and a protein from Arabidopsis, AT1g60070, whose amino acid sequences are 14 to $16 \%$ identical to P3. Amino acid alignments of the P3 C-terminal region containing the two mutations responsible for breaking Rsv4 resistance showed that amino acid $\mathrm{K}$ at position 1,033 is conserved among all AP-1 homologs (Fig. 5). However, amino acid $\mathrm{G}$, not $\mathrm{R}$, at position 1,054 is conserved in plant AP-1-like proteins (Fig. 5). Modeling of P3 of isolates L, L-RB, and NP-L demonstrated that the predicted structure is essentially the same. However, either Q1033K or G1054R mutation did cause local structural modifications at the surface (Supplementary Fig. S2).

\section{DISCUSSION}

In a previous study, we reported the identification of a naturally occurring SMV G2 isolate, L-RB, which overcomes $R s v 4$ resistance and its closely related nonresistance-breaking isolate, L (Gagarinova et al. 2008a). L-RB is likely derived from variants of $\mathrm{L}$ generated during its error-prone replication catalyzed by the virus-encoded RNA-dependent RNA polymerase (Gagarinova et al. 2008a and b). In this study, we constructed SMV infectious clones to investigate what genetic determinants are responsible for virulence on $R s v 4$ soybean. Our swapping experiments mapped the virulence determinant to the $\mathrm{M}$ fragment of L-RB (Figs. 1-3). Subsequent site-directed mutagenesis pin-pointed the Q1033K mutation in the $\mathrm{P} 3$ protein rather than the other three substitutions (two in P3 and one in CI) that plays an essential role in converting avirulence to virulence (Fig. 4). Because all the three different amino acids in P3 are located downstream of P3N-PIPO, a product of translational frameshift within P3 (Chung et al. 2008; Wei et al. 2010b), P3N-PIPO seems not to be involved in overcoming the Rsv4 resistance in this study. Thus, P3 is an elicitor for Rsv4-mediated resistance.

The Turnip mosaic virus (TuMV) P3 protein also functions as an avirulent determinant in TuRBO3 or TuRBO4 brassicas (Jenner et al. 2002, 2003). A single amino acid mutation destroys its elicitor function. More recently, TuMV P3 has been shown to determine the systemic necrosis in Arabidopsis through the gene-for-gene interaction between TuNI (TuMV necrosis inducer) and P3 (Kim et al. 2010). The elicitor function of P3 was previously suggested for the SMV G7 strain that induces lethal systemic hypersensitive response on the $R s v 1$-genotype soybean (Hajimorad et al. 2005). In G2-Rsvl interactions, absence of P3 elicitor function alone is not sufficient for conferring virulence (Hajimorad et al. 2006) and HCPro complementation of $\mathrm{P} 3$ is required for $\mathrm{G} 2$ to gain virulence (Hajimorad et al. 2008). It has been suggested that a polypeptide containing HC-Pro and P3 acts as the SMV elicitor of Rsv1 (Hajimorad et al. 2008). This assumption is in contradiction to the fact that HC-Pro is a papain-like cysteine proteinase that highly efficiently autocleaves the polyprotein at the junction of its $\mathrm{C}$ terminus and the $\mathrm{N}$ terminus of $\mathrm{P} 3$ (Carrington et al. 1989). Thus, it is more likely that HC-Pro and P3 may function redundantly to coordinate virulence toward Rsvl resistance (Hajimorad et al. 2008). Alternatively, P3 may act as an elicitor whereas HC-Pro may serve as a suppressor of the Rsv1-mediated responses, as suggested by Hajimorad and associates (2008). In this study, we found that P3 alone can function as an avirulence elicitor of Rsv4. As we mentioned earlier, unlike $R s v 1$, the $R s v 4$-mediated resistant response is not involved in hypersensitive response (Gunduz et al. 2004). It is tempting to speculate that HC-Pro participates in the $R s v 1$-mediated hypersensitive response. If so, the effects of HC-Pro mutants essential for the Rsv 1 virulence on Rsv 4 resistance are worth evaluation. This should help advance our understanding of complex interactions between different SMV strains and $R$ genes.

To further investigate G2-Rsv4 interactions, an experimental evolutionary approach was used to identify new Rsv4 resistance-breaking isolates. The P3 protein of all six new resistance-breaking isolates contains either Q1033K or G1054R mutations. The G1054R mutation was also confirmed to be sufficient for overcoming $R s v 4$ resistance. These data again support the idea that P3 alone plays an elicitor role in G2Rsv4 interactions. Because the Rsv4 gene also confers resistance to G7 isolates, it would be interesting to determine whether G7 chimeras containing P3 of L-RB or G7 mutants containing either the Q1033K or G1054R mutation become virulent on $R s v 4$ soybean.

P3 is a functionally poorly characterized potyviral protein. In addition to its elicitor role, it may have diverse functions such as virus replication, systemic infection, pathogenicity, and movement (Chu et al. 1997; Cui et al. 2010; Eggenberger et al. 2008; Hajimorad et al. 2008; Jenner et al. 2003; Johansen et al. 2001; Klein et al. 1994; Merits et al. 1999). In this study, we show that two L mutants in which P3 has double amino acid substitutions (Q1033K and I1081V) or triple mutations (Q1033K, I1081V, and N1171S) lost infectivity on the susceptible indicator soybean (Fig. 4). It is possible that these mutations in P3 may affect the local structural conformation that is essential for its functioning in the infection process. In a parallel study, we tagged P3 with a fluorescent protein and found that the recombinant virus is lethal, too (R. V. Chowda-Reddy and A. Wang, unpublished data). The data presented in this study represent the first genetic evidence that P3 is required in potyvirus infection, consistent with our recent finding that P3 colocalizes with the virus replication vesicles (Cui et al. 2010). However, the exact role of P3 in SMV life cycles remains to be investigated.

The structure of an avirulent elicitor is essential for recognition by the plant resistance factor (Janzac et al. 2010). The P3 protein contains two hydrophobic domains located at the $\mathrm{N}$ and $\mathrm{C}$ terminus, respectively. The Q1033K mutation is located within the C-terminal membrane domain and the G1054R mutation is near this domain. The three-dimensional structure modeling of P3 and its Q1033K and G1054R variants suggests that they are structurally similar overall but have local structural differences. In Q1033K and G1054R mutations, K and R are two positively charged amino acids. Amino acids K (after mutation) and $\mathrm{G}$ (before mutation) are conserved in the plant AP-1-like proteins (Fig. 5). It is possible that these two mutations, with positively charged amino acids in P3 of the resistance-breaking isolates, disturb the interaction of P3 with its host acceptor, leading to the disruption of the Rsv4-mediated resistance signaling pathway. Because none of soybean SMV $R$ genes has been cloned and sequenced, it is not clear whether P3 directly interacts with the Rsv4-encoded protein. This is one of the areas of our future study.

In summary, our results show that P3 of the SMV G2 isolates is an avirulent determinant for Rsv4. The Rsv4-mediated resistance seems very fragile because single-nucleotide mutations in the P3 cistron may lead to the occurrence of new 
breaking isolates. Considering that high genetic diversity of SMV that has been reported and new variants will be expected, there is a high risk associated with the utilization of soybean cultivars that carry a single resistance gene. This is supported by the identification of $R s v$ resistance-breaking SMV isolates (Choi et al. 2005; Gagarinova et al. 2008a). A better understanding of molecular SMV $-R$ gene interactions will certainly help in developing new strategies for durable SMV resistance.

\section{MATERIALS AND METHODS}

\section{SMV isolates, soybean cultivars, and virus detection.}

SMV isolates L and L-RB obtained previously (Gagarinova et al. 2008a), their derived infectious clones constructed in this study (see below), chimeric clones, and mutants were used in this study. Soybean (Glycine max) cvs. William 82 (susceptible host), PI 96983 (Rsv1), L78-379 (Rsv1), L-29 (Rsv3), Suweon $97(R s v 1-h)$, and V94-5152 (Rsv4) were grown in a growth chamber with $16 \mathrm{~h}$ of light at $22^{\circ} \mathrm{C}$ and $8 \mathrm{~h}$ of darkness at $18^{\circ} \mathrm{C}$. For SMV inoculation, mechanical inoculation with infected tissues as an inoculum and biolistic bombardment with SMV infectious plasmid DNA were carried out following previous publications (Gagarinova et al. 2008a; Hajimorad and Hill 2001). Virus detection was carried out by ELISA and RTPCR as described (Gagarinova et al. 2008a).

\section{Construction of infective clones pSMV-L and pSMV-LRB and their chimeric clones.}

Total RNA isolated from soybean plants infected by SMV L and L-RB isolates (Gagarinova et al. 2008a) was reverse-transcribed into cDNA and them amplified by PCR to generate three cDNA fragments: N, M, and C (Fig. 1). The cloning strategy was described previously (Hajimorad et al. 2003; Wang et al. 2006). The three fragments were sequentially cloned into a modified pBR322 containing the $35 \mathrm{~S}$ promoter and the NOS terminator to generate SMV full-length clones pSMV-L and pSMV-LRB. The sequence of inserted cDNA was verified against published sequences of $\mathrm{L}$ and L-RB (EU871724 and EU871725) by sequencing. The infectivity and pathogenicity of pSMV-L and pSMV-LRB were confirmed by biolistic introduction of the two clones into soybean cultivars described above. Six chimeric clones (Fig. 3) -that is, pSMV-L $\left(\mathrm{N}_{\mathrm{LRB}}\right)$, pSMV-L $\left(\mathrm{M}_{\mathrm{LRB}}\right)$, pSMV-L $\left(\mathrm{C}_{\mathrm{LRB}}\right)$, pSMV$\operatorname{LRB}\left(\mathrm{N}_{\mathrm{L}}\right)$, pSMV-LRB $\left(\mathrm{M}_{\mathrm{L}}\right)$, and pSMV-LRB $\left(\mathrm{C}_{\mathrm{L}}\right)$-were obtained by swapping of $\mathrm{N}, \mathrm{M}$, and $\mathrm{C}$ fragments through digestion with the enzymes indicated (Fig. 1) and ligation following standard protocols. The resulting swapping clones were verified by restriction analysis and sequencing. For the infectivity test, each construct was biolistically introduced into three 2week-old Williams 82 ( $r s v)$ seedlings. Infected leaves were harvested 15 days postbombardment and stored in a $-80^{\circ} \mathrm{C}$ freezer for the pathogenicity test. The pathogenicity test was repeated three times and, each time, two 2-week-old $r s v$ seedlings (Williams 82) and $20 R s v$ soybean plants (4 for each of five cultivars described above) were mechanically inoculated as previously described (Gagarinova et al. 2008a).

\section{Mutagenesis of the $M$ fragment.}

The $\mathrm{M}$ fragment contains four amino acid differences between SMV L and L-RB (Fig. 1). For the convenience of mutagenesis, the $\mathrm{M}$ fragment of pSMV-L and pSMV-LRB was taken out by digestion and cloned into the TOPO plasmid (Invitrogen, Carlsbad, CA, U.S.A.). The resulting clones served as a template to introduce single, double, and triple mutations into the $\mathrm{M}$ fragment using a QuickChange SiteDirected Mutagenesis kit (Stratagene, La Jolla, CA, U.S.A.) following the supplier's instructions. The M fragment contain- ing the desired mutations was confirmed by sequencing and then inserted back into pSMV-L or pSMV-LRB to produce mutant clones (Fig. 4). All the M-fragment mutant clones were verified by restriction analyses and sequencing. Each of these mutant clones were bombarded onto three Williams 82 soybean seedlings as described above. At 15 days postbombardment, the infected leaves were used as inoculum for pathogenicity tests with different soybean cultivars described above. For the two P3 mutants that lost infectivity, six experiments were performed with three Williams 82 seedlings for each experiment, and none of seedlings showed symptoms or positive with ELIA or RT-PCR.

\section{Isolation and sequencing \\ of new $R s v 4$ resistance-breaking isolates.}

Williams 82 soybean plants were inoculated with all nine infectious clones (Figs. 3 and 4) that were unable to infect V94-5152. At 5 weeks postinoculation, the infected young leaves were used as inocula. After another passage on Williams 82 , the infected leaf tissues were mechanically inoculated into V94-5152. Six V94-5152 soybean plants showed systemic symptoms. The $M$ region of all six isolates was sequenced. Two representative new resistance-breaking isolates, NP-L derived from $\mathrm{pSMV}-\mathrm{L}$ and NP-C-L from $\mathrm{pSMV}-\mathrm{L}\left(\mathrm{C}_{\mathrm{LRB}}\right)$, were completely sequenced.

\section{Modeling and construction of the structure of $\mathrm{P3}$ and its variants.}

ConSeq version 1.1 (Berezin et al. 2004) was used to score for the putative functional and structural important residues. HHpred (Biegert et al. 2006) and PHYRE (Kelley and Sternberg 2009) servers were used for protein secondary structure prediction and PDB homolog search. Because no perfect PDB homologs were available, PDB template $1 \mathrm{w} 63: \mathrm{K}$, showing highest similarity with P3, was used as a template. Modeling of the structures of P3 and its variants was carried out with the I-TASSER server (Zhang 2008) and the P3 structure was constructed by PyMOL.

\section{ACKNOWLEDGMENTS}

We thank J. Hill (Iowa State University) for helpful advice and comments on the manuscript, J. McNeil (Agriculture and Agri-Food Canada [AAFC]) for expert technical assistance, I. van Grinsven (AAFC) for DNA sequencing, and A. Molnar (AAFC) for photography. This work was supported in part by AAFC Canadian Crop Genomics Initiative, the Natural Sciences and Engineering Research Council of Canada, and Ontario Soybean Growers.

\section{LITERATURE CITED}

Berezin, C., Glaser, F., Rosenberg, Y., Paz, I., Pupko, T., Fariselli, P., Casadio, R., and Ben-Tal, N. 2004. ConSeq: the identification of functionally and structurally important residues in protein sequences. Bioinformatics 20:1322-1324.

Berger, P. H., Adams, M. J., Barnett, O. W., Brunt, A. A., Hammond, J., Hill, J. H., Jordan, R. L., Kashiwazaki, S., Rybicki, E., Spence, N., Stenger, D. C., Ohki, S. T., Uyeda, I., van Zaayen, A., Valkonen, J., and. Vetten, H. J. 2005. Potyvirida. Pages 819e-841e in: Virus Taxonomy. Eighth Report of the International Committee on Taxonomy of Viruses. C. M. Fauquet, M. A. Mayo, J. Maniloff, U. Desselbergar, and L. A. Ball, eds. Elsevier Academic Press, San Diego, CA, U.S.A.

Biegert, A., Mayer, C., Remmert, M., Söding, J., and Lupas, A. N. 2006 The MPI bioinformatics toolkit for protein sequence analysis. Nucleic Acid Res. 34:335-339.

Buss, G. R., Ma, G., Kristipati, S., Chen, P., and Tolin, S. A. 1999. A new allele at the Rsv3 locus for resistance to soybean mosaic virus. Page 490 in: Proc. World Soybean Res. Conf. VI. H. E. Kauffman, ed. Superior Printing, Champaign, IL, U.S.A.

Carrington, J. C., Freed, D. D., and Sanders, T. C. 1989. Autocatalytic processing of the potyvirus helper component proteinase in Escherichia 
coli and in vitro. J. Virol. 63:4459-4463.

Cho, E. K., and Goodman, R. M. 1979. Strains of soybean mosaic virus: classification based on virulence in resistant soybean cultivars. Phytopathology 69:467-470.

Choi, B. K., Koo, J. M., Ahn, H. J., Yum, H. J., Choi, C. W., Ryu, K. H., Chen, P., and Tolin, S. A. 2005. Emergence of $R s v$-resistance breaking soybean mosaic virus isolates from Korean soybean cultivars. Virus Res. 112:42-51.

Chu, M., Lopez-Moya, J. J., Llave-Correas, C., and Pirone, T. P. 1997. Two separate regions in the genome of the tobacco etch virus contain determinants of the wilting response of Tabasco pepper. Mol. PlantMicrobe Interact. 10:472-480.

Chung, B. Y., Miller, W. A., Atkins, J. F., and Firth, A. E. 2008. An overlapping essential gene in the Potyviridae, Proc. Natl. Acad. Sci. U.S.A. 105:5897-5902.

Cui, X., Wei, T., Chowda-Reddy, R. V., Sun, G., and Wang, A. 2010. The Tobacco etch virus $\mathrm{P} 3$ protein forms mobile inclusions via the early secretory pathway and traffics along actin microfilaments. Virology 397:56-63.

Eggenberger, A. L., Hajimorad, M. R., and Hill, J. H. 2008. Gain of virulence on Rsvl-genotype soybean by an avirulent Soybean mosaic virus requires concurrent mutations in both P3 and HC-Pro. Mol. Plant-Microbe Interact. 21:931-936.

Eiamtanasate, S., Juricek, M., and Yap, Y. K., 2007. C-terminal hydrophobic region leads PRSV P3 protein to endoplasmic reticulum. Virus Genes 35:611-617.

Gagarinova, A. G., Babu, M. R., Poysa, V., Hill, J. H., and Wang, A 2008a. Identification and molecular characterization of two naturally occurring Soybean mosaic virus isolates that are closely related but differ in their ability to overcome $R s v 4$ resistance. Virus Res.138:50-56.

Gagarinova, A. G, Babu, M., Strömvik, M. V., and Wang, A. 2008b. Recombination analysis of Soybean mosaic virus sequence reveals evidence of RNA recombination between distinct pathotypes. Virol. J. $5: 143$

García-Arenal, F., Fraile, A., and Malpica, J. M. 2003. Variation and evolution of plant virus population. Int. Microbiol. 6:225-232.

Gore, M. A., Hayes, A. J., Jeong, S. C., Yu, Y. G., Buss, G. R., and Saghai Maroof, M. A. 2002. Mapping tightly linked genes controlling potyvirus infection at the Rsv1 and Rpv1 region in soybean. Genome 45:592599.

Gunduz, I., Buss, G. R., Ma, G., Chen, P., and Tolin, S. A. 2001. Genetic analysis of resistance to soybean mosaic virus in OX670 and Harosoy soybean. Crop Sci. 41:1785-1791.

Gunduz, I., Buss, G. R., Chen, P., and Tolin, S. A. 2002. Characterization of SMV resistance genes in Tousan 140 and Hourei soybean. Crop Sci. 42:90-95.

Gunduz, I., Buss, G. R., Ma, G., Chen, P., and Tolin, S. A. 2004. Genetic and phenotypic analysis of Soybean mosaic virus resistance in PI 88788 soybean. Phytopathology 94:687-692.

Hajimorad, M. R., and Hill, J. H. 2001. Rsv1-mediated resistance against Soybean mosaic virus $-\mathrm{N}$ is hypersensitive response-independent at inoculation site, but has the potential to initiate a hypersensitive responselike mechanism. Mol. Plant-Microbe Interact. 14:587-598.

Hajimorad, M. R., Eggenberger, A. L., and Hill, J. H. 2003. Evolution of Soybean mosaic virus-G7 molecularly cloned genome in Rsv1-genotype soybean results in emergence of a mutant capable of evading Rsv1mediated recognition. Virology 314:497-509.

Hajimorad, M. R., Eggenberger, A. L., and Hill, J. H. 2005. Loss and gain of elicitor function of Soybean mosaic virus G7 provoking Rsv1-mediated lethal systemic hypersensitive response maps to P3. J. Virol. 79:1215-1222.

Hajimorad, M. R., Eggenberger, A. L., and Hill, J. H. 2006. Strain-specific P3 of Soybean mosaic virus elicits Rsv1-mediated extreme resistance, but absence of P3 elicitor function alone is insufficient for virulence on Rsv1-genotype soybean. Virology 345:156-166.

Hajimorad, M. R., Eggenberger, A. L., and Hill, J. H. 2008. Adaptation of Soybean mosaic virus avirulent chimeras containing P3 sequences from virulent strains to Rsv1-genotype soybeans is mediated by mutations in HC-Pro. Mol. Plant-Microbe Interact. 21:937-946.

Hayes, A. J., Ma, G., Buss, G. R., and Saghai Maroof, M. A. 2000. Molecular marker mapping of $R s v 4$, a gene conferring resistance to all known strains of Soybean mosaic virus. Crop Sci. 40:1434-437.

Janzac, B., Montarry, J., Palloix, A., Navaud, O., and Moury, B. 2010. A point mutation in the polymerase of Potato virus $Y$ confers virulence towards the Pvr4 resistance of pepper and a high competitiveness cost in susceptible cultivar. Mol. Plant-Microbe Interact. 23:823-830.
Jayaram, C. H., Hill, J. H., and Miller, W. A. 1992. Complete nucleotide sequences of two Soybean mosaic virus strains differentiated by response of soybean containing the Rsv resistance gene. J. Gen. Virol. 73:2067-2077.

Jenner, C. E., Tomimura, K., Ohshima, K., Hughes, S. L., and Walsh, J. A. 2002. Mutations in Turnip mosaic virus P3 and cylindrical inclusion proteins are separately required to overcome two Brassica napus resistance genes. Virology 300:50-59.

Jenner, C. E., Wang, X., Tomimura, K., Ohshima, K., Ponz, F., and Walsh, J. A. 2003. The dual role of the potyvirus P3 protein of Turnip mosaic virus as a symptom and avirulence determinant in brassicas. Mol. PlantMicrobe Interact. 16:777-784.

Jeong, S. C., Kristipati, S., Hayes, A. J., Maughan, P. J., Noffsinger, S. L., Gunduz, I., Buss, G. R., and Saghai Maroof, M. A. 2002. Genetic and sequence analysis of markers tightly linked to the Soybean mosaic virus resistance gene, $R s v 3$. Crop Sci. 42:265-270.

Johansen, I. E., Lund, O. S., Hjulsager, C. K., and Laursen, J. 2001. Recessive resistance in Pisum sativum and potyvirus pathotype resolved in a gene for cistron correspondence between host and virus. J. Virol. 75:6614-6669.

Kelly, L. A., and Sternberg, M. J. E. 2009. Protein structure prediction on the Web: a case study using the Phyre server. Nat. Protocols 4:363-371.

Kim, B. M., Suehiro, N., Natsuaki, T., Inukai, T., and Masuta, C. 2010 The P3 protein of Turnip mosaic virus can alone induce hypersensitive response-like cell death in Arabidopsis thaliana carrying TuNI. Mol. Plant-Microbe Interact. 23:144-152.

Klein, P. G., Klein, R. R., Rodríguez-Cerezo, E., Hunt, A. G., and Shaw, J. G. 1994. Mutational analysis of the tobacco vein mottling virus genome. Virology 204:759-769.

Liao, L., Chen, P., Buss, G. R., Yang, Q., and Tolin, S. A. 2002. Inheritance and allelism of resistance to soybean mosaic virus in Zao 18 soybean from China. J. Hered. 93:447-452.

Maule, A. T., Caranta, C., and Boulton, M. I. 2007. Sources of natural resistance to plant viruses: status and prospects. Mol. Plant Pathol. $8: 223-231$

Merits, A., Guo, D., Järvekülg, L., and Saarma, M. 1999. Biochemical and genetic evidence for interactions between potato A potyvirus-encoded proteins $\mathrm{P} 1$ and $\mathrm{P} 3$ and proteins of the putative replication complex. Virology 263:15-22.

Restrepo-Hartwig. M. A., and Carrington, J. C. 1994. The tobacco etch Potyvirus 6-kilodalton protein is membrane associated and involved in viral replication. J. Virol. 68:2388-2397.

Schaad, M. C., Jensen, P. E., and Carrington, J. C. 1997. Formation of plant RNA virus replication complexes on membranes: role of an endoplasmic reticulum-targeted viral protein. EMBO (Eur. Mol. Biol. Organ.) J. 16:4049-4059.

Urcuqui-Inchima, S., Haenni, A. L., and Bernardi, F. 2001. Potyvirus proteins: a wealth of functions. Virus Res. 74:157-175.

Wang, L., Eggenberger, A., Hill, J., and Bogdanove, A. J. 2006. Pseudomonas syringae effector $a v r B$ confers soybean cultivar-specific avirulence on Soybean mosaic virus adapted for transgene expression but effector avrPto does not. Mol. Plant-Microbe Interact. 19:304-312.

Wei, T., and Wang, A. 2008. Biogenesis of cytoplasmic membranous vesicles for plant potyvirus replication occurs at endoplasmic reticulum exit sites in a COPI- and COPII-dependent manner. J. Virol. 82:1225212264.

Wei, T., Huang, T.-S., McNeil, J. Laliberté, J.-F, Hong, J., Nelson, R. S. and Wang, A. 2010a. Sequential recruitment of the endoplasmic reticulum and chloroplasts for plant potyvirus replication. J. Virol. 84:799809.

Wei, T., Zhang, C., Hong, J., Xiong, R., Kasschau, K. D., Zhou, X., Carrington, J. C., and Wang, A. 2010b. Formation of complexes at plasmodesmata for potyvirus intercellular movement is mediated by the viral protein P3N-PIPO. PLoS Pathog. 6(6):e1000962.

Zhang, Y. 2008. I-TASSER server for protein 3D structure prediction. BMC Bioinf. 9:40.

Zheng, C., Chen, P., and Gergerich, R. 2005. Characterization of resistance to Soybean mosaic virus in diverse soybean germplasm. Crop Sci. 45:2503-2509.

\section{AUTHOR-RECOMMENDED INTERNET RESOURCES}

Phytozome tool for green plant comparative genomics: www.phytozome.net/index.php

PyMOL open-source molecular graphic tool: www.pymol.org 